\title{
On Types over von Neumann Subalgebras and the Dye Correspondence
}

\author{
By \\ Marie CHODA
}

The family of abelian projections of von Neumann algebras plays an important role in the theory of types (discrete and continuous). An abelian projection $E$ of a von Neumann algebra $\mathscr{A}$ is defined by the requirement that each projection $P$ in $\mathscr{A}$ dominated by $E$ is written as $P=Q E$ for some $Q$ in the center $\mathscr{Z}$ of $\mathscr{A}$. We have taken an interest in generalizing this definition of an abelian projection by using a general von Neumann subalgebra of $\mathscr{A}$ in place of the center $\mathscr{Z}$.

In $[1]$, we defined a projection abelian over a von Neumann subalgebra (see Definition 3 below) and proved that some elementary properties of abelian projections are preserved under the generalization. Those results lead us to a natural generalization of continuous von Neumann algebras. In [2], we have extended the definition of continuous von Neumann algebras (Definition 10 below) and proved that a von Neumann subalgebra $\mathscr{B}$ of a von Neumann algebra $\mathscr{A}$ contained in the center has a useful property relative to an expectation (that is, $\mathscr{B}$ is a strong Maharam subalgebra of $\mathscr{A}$ in the sense of Definition 15) if $\mathscr{A}$ is continuous over $\mathscr{B}$ (Theorem A).

In this note, we shall define a von Neumann algebra $\mathscr{A}$ discrete over a von Neumann subalgebra $\mathscr{B}$. We prove that $\mathscr{A}$ splits into the direct sum of the part continuous over $\mathscr{B}$ and the part discrete over $\mathscr{B}$ if $\mathscr{B}$ is contained in the center of $\mathscr{A}$ (Theorem 14 in $\S 2$ ).

When a von Neumann subalgebra $\mathscr{B}$ of a von Neumann algebra $\mathscr{A}$ satisfies some conditions, $\mathscr{B}$ is discrete (resp. continuous) over the center of $\mathscr{A}$ if and only if $\mathscr{A}$ is discrete (resp. continuous) in the usual sense (Theorem 22 in $\S 3$ ).

Communicated by H. Araki, September 18, 1972. 
In $\S 4$, we shall apply theorems in $\S 3$ to the crossed product $G \otimes \mathscr{A}$ of a von Neumann algebra $\mathscr{A}$ by a freely acting automorphism group $G$ of $\mathscr{A}$ with the following results:

One of main results in Dye's paper [5] is that if $\mathscr{A}$ is a non-atomic abelian von Neumann algebra, then there is a $1: 1$ correspondence between full subgroups of the full group $[G]$ determined by $G$ and intermediate von Neumann subalgebras of $G \otimes \mathscr{A}$. Haga-Takeda have extended, in their paper $[7]$, this result to a $\sigma$-finite finite von Neumann algebra $\mathscr{A}$. On the other hand, Dye has introduced the types (I and II) for a freely acting automorphism group on an abelian von Neumann algebra, and shown that the correspondence in the above conserves the type; if an intermediate von Neumann algebra is discrete (resp. continuous), then the corresponding subgroup is of type I (resp. type II).

We shall define types (discrete type and continuous type) for automorphism groups of von Neumann algebras in Definition 23. This definition is a generalization of that due to Dye. And we shall show that the correspondence of intermediate von Neumann subalgebras of $G \otimes \mathscr{A}$ and full subgroups of the full group $[G]$ determined by $G$, due to Haga-Takeda, preserves the type in the sense of Dye (Theorem 24 in \$4).

The author would like to express her appreciation to Prof. Y. Haga and Prof. Z. Takeda for giving her the opportunity to see pre-publication copy of their paper, to which she is indebted deeply, and to Prof. Y. Nakagami for taking the pains reading her manuscript carefully and giving her valuable comments.

We shall use the terminologies and notations due to Dixmier [3] throughout this note without further explanations.

2. In the sequel, let $\mathscr{A}$ be a von Neumann algebra and $\mathscr{B}$ a von Neumann subalgebra of $\mathscr{A}$. Denote by $\mathscr{B}^{c}$ the relative commutant $\mathscr{B}^{\prime} \cap \mathscr{A}$ of $\mathscr{B}$ in $\mathscr{A}, \mathscr{B}^{D}$ the set of all projections of $\mathscr{B}$ and $\bar{E}$ the $\mathscr{B}$-support of $E \in \mathscr{A}^{p}$, that is,

$$
\bar{E}=\inf \left\{P \in \mathscr{B}^{p} ; P \geqq E\right\} .
$$

Lemma 1. Let $\left(E_{\iota}\right)_{\iota \in I}$ be a family of projections in $\mathscr{A}$, then

$$
\overline{\sup _{\iota} E_{\iota}}=\sup _{\iota} \overline{E_{\iota}} \text {. }
$$


Proof. By the definition, $\sup _{\iota} \overline{E_{\iota}} \geqq \sup _{\iota} E_{\iota}$, so $\sup _{\iota} \overline{E_{\imath}} \geqq \overline{\sup _{\iota} E_{\iota}}$. Conversely $\overline{\sup _{\iota} E_{\iota}} \geqq \overline{E_{\iota}}$, which implies that $\overline{\sup _{\iota} E_{\iota}} \geqq \sup _{\iota} \overline{E_{\iota}}$. Therefore $\overline{\sup _{\iota} E_{\iota}}=\sup _{\iota} \overline{E_{\iota}}$.

For completeness we include a proof of the following Lemma, which is proved in [1].

Lemma 2. If $\bar{E} P$ is a projection for $E \in\left(\mathscr{B}^{c}\right)^{p}$ and $P \in \mathscr{B}$, then

$$
\overline{E P}=\bar{E} P .
$$

Proof. $\overline{E P} \leqq \bar{E} P$ is clear by the definition. If $\overline{E P} \neq \bar{E} P$, then there exist two projections $Q$ and $R$ in $\mathscr{B}$ such that

$$
\bar{E} P \supsetneqq Q \geqq E P
$$

and that

$$
\bar{E}(I-P) \geqq R \geqq E(I-P) .
$$

Therefore $Q+R \in \mathscr{B}^{p}$ satisfies $\bar{E} ¥ Q+R \geqq E$, which contradicts the definition of $\bar{E}$.

The following definition is introduced in [1] as a generalization of the notion of abelian projections.

Definition 3. A projection $E \in \mathscr{A}$ is called abelian over $\mathscr{B}$ if $E \in \mathscr{B}^{c}$ and for every projection $P \in \mathscr{A}$ with $P \leqq E$, there exists a projection $Q \in \mathscr{B}$ such that $P=Q E$.

Lemma 4. Let $E$ be a projection in $\mathscr{A}$ abelian over $\mathscr{B}$. A projection $F$ in $\mathscr{B}^{c}$ is abelian over $\mathscr{B}$ if $F \leqq E$.

Proof. Let $P$ be a projection in $\mathscr{A}$ with $P \leqq F$. Then there exists a $Q \in \mathscr{B}^{p}$ such that $P=Q E$ because $P \leqq E$ and $E$ is abelian over $\mathscr{B}$. Hence

$$
P=P F=Q E F=Q F
$$

For a general form of Lemma 4, see [1; Lemma 3].

The following lemma, proved in [2], gives an alternative algebraic definition of projections abelian over $\mathscr{B}$.

Lemma 5. $E \in \mathscr{A}^{p}$ is abelian over $\mathscr{B}$ if and only if $E \in \mathscr{B}^{c}$ and 
$\mathscr{A}_{E}=\mathscr{B}_{E}$.

Now, we shall extend the definition of discrete von Neumann algebras as the following:

Definition 6. A von Neumann algebra $\mathscr{A}$ is called discrete over $\mathscr{B}$ if there exists an $E \in \mathscr{A}^{p}$ which is abelian over $\mathscr{B}$ and $\bar{E}=I$.

Remark. Let $\mathscr{Z}$ be the center of a von Neumann algebra $\mathscr{A}$. Then if $\mathscr{A}$ is discrete over $\mathscr{Z}, \mathscr{A}$ is discrete in the usual sense cf. [3]. If $\mathscr{A}$ is an abelian von Neumann algebra and discrete over $\mathscr{B}$, then $\mathscr{B}$ is called by Dye [4], a type I subalgebra; cf. Corollary 12 below.

Example. Let $\mathscr{B}$ be a von Neumann algebra and $\mathscr{C}$ a discrete factor, then $\mathscr{B} \otimes \mathscr{C}$ is discrete over $\mathscr{B} \otimes I$.

In fact, let $P$ be a minimal projection in $\mathscr{C}$. Clearly $I \otimes P$ is abelian over $\mathscr{B} \otimes I$ and $\mathscr{B} \otimes I$-support of $I \otimes P=I \otimes I$. Therefore, $\mathscr{B} \otimes \mathscr{C}$ is discrete over $\mathscr{B} \otimes I$.

Theorem 7. Let $\mathscr{B}$ be a von Neumann subalgebra of $\mathscr{A}$ contained in the center $\mathscr{Z}$ of $\mathscr{A}$. Then $\mathscr{A}$ is discrete over $\mathscr{B}$ if and only if each nonzero projection in $\mathscr{B}$ dominates a nonzero projection abelian over $\mathscr{B}$.

Proof. If each nonzero projection in $\mathscr{B}$ dominates a nonzero projection abelian over $\mathscr{B}$, by Zorn's lemma, we have a maximal orthogonal family $\left(G_{\iota}\right)_{\iota \in I}$ of nonzero projections in $\mathscr{B}$ satisfying the following properties: for any $\iota \in I$, there exists an $E_{\iota} \in \mathscr{A}^{p}$ which is abelian over $\mathscr{B}$ and $\bar{E}_{\iota}=G_{\iota}$. Put $G=\Sigma_{\iota} G_{\iota}$. If $G \neq I$, there exists a nonzero $F \in \mathscr{A}^{p}$ which is abelian over $\mathscr{B}$ and $F \leqq I-G$ because $I-G \in \mathscr{B}$. Since $\bar{F} \leqq I-G, \bar{F}$ is orthogonal to each $G_{\iota}$, which contradicts the maximality of $\left(G_{\iota}\right)_{\iota \in I}$. Therefore, we have $G=I$. Put $E=\Sigma_{\iota} E_{\iota}$. Since $\left(E_{\iota}\right)_{\iota \in I}$ is an orthogonal family, $\bar{E}=$ $\overline{\Sigma_{\iota} E_{\iota}}=\Sigma_{\iota} G_{\iota}=G=I$ by Lemma 1 . On the other hand $\mathscr{A}_{E}=\Sigma_{\iota} \oplus \mathscr{B}_{E_{\iota}}=\mathscr{B}_{E}$ because $E_{\iota}$ is abelian over $\mathscr{B},\left(G_{\iota}\right)_{\iota} \in I$ is an orthogonal family of projections in $\mathscr{B}$ with $\sum_{\iota} G_{\iota}=I$ and $\mathscr{B}$ is contained in the center of $\mathscr{A}$ (cf. [3. p.22]). Hence $E$ is abelian over $\mathscr{B}$. Thus there exists an $E \in \mathscr{A}^{p}$ which is abelian over $\mathscr{B}$ and $\vec{E}=I$.

Conversely, suppose that there exists a projection $E$ which is abelian over $\mathscr{B}$ and $\bar{E}=I$. For a nonzero $P \in \mathscr{B}^{p}$, put $Q=E P$. Then $Q \leqq P$ and 
$\bar{Q}=\overline{E P}=\bar{E} P=P \neq 0$ by Lemma 2 , so $Q \neq 0$. Since $\mathscr{B}$ is contained in the center of $\mathscr{A}, Q \in \mathscr{B}^{c}$. Therefore, by Lemma $4, Q$ is abelian over $\mathscr{B}$. Thus each nonzero projection in $\mathscr{B}$ dominates a nonzero projection abelian over $\mathscr{B}$.

Proposition 8. Let $\mathscr{B}$ be a von Neumann subalgebra of a von Neumann algebra $\mathscr{A}$ containing the center of $\mathscr{A}$. Assume that $\mathscr{A}$ is discrete over $\mathscr{B}$.

(1) $\mathscr{A}$ is discrete if and only if $\mathscr{B}$ is discrete;

(2) $\mathscr{A}$ is continuous if and only if $\mathscr{B}$ is continuous;

(3) $\mathscr{A}$ is a factor if and only if $\mathscr{B}$ is a factor;

(4) If $\mathscr{A}$ is a hyperfinite factor, then $\mathscr{B}$ is a hyperfinite factor.

Proof. If $\mathscr{A}$ is discrete over $\mathscr{B}$, there exists an $E \in\left(\mathscr{B}^{c}\right)^{p}$ with $\mathscr{A}_{E}$ $=\mathscr{B}_{E}$ and $\bar{E}=I$. Since $E \in \mathscr{B}^{\prime}$ and the $\mathscr{B} \cap \mathscr{B}^{\prime}$-support of $E=I$, it follows that $\mathscr{B} \cong \mathscr{B}_{E}=\mathscr{A}_{E}$ [3. p. 19, Prop. 2]. Therefore, (4) and the "only if", parts of (1), (2) and (3) are obvious (see, for instance [3]). Conversely, $\mathscr{A}^{\prime} \cong \mathscr{A}_{E}^{\prime}=\mathscr{B}_{E}^{\prime}$ because $E \in \mathscr{A}$ and the central support of $E=I$ by $\mathscr{B} \supset$ the center of $\mathscr{A}$. If $\mathscr{B}$ satisfies the condition of (1) (2) (3) respectively, then $\mathscr{B}^{\prime}$ does so (see, for instance [3]). Therefore $\mathscr{A}$ does so.

Proposition 9. Let $\mathscr{A}$ be a $\sigma$-finite infinite factor. If $\mathscr{A}$ is discrete over $\mathscr{B}$, then $\mathscr{A}$ is isomorphic to $\mathscr{B} \otimes \mathscr{L}(\Re)$ for some Hilbert space $\Re$, where $\mathscr{L}(\Re)$ is the factor of all bounded operators on $\Re$.

Proof. If $\mathscr{A}$ is discrete over $\mathscr{B}$, there exists an $E \in\left(\mathscr{B}^{c}\right)^{p}$ with $\mathscr{A}_{E}=$ $\mathscr{B}_{E}$ and $\bar{E}=I$. Since $\mathscr{A}$ is a $\sigma$-finite infinite factor, it follows that $\mathscr{A}$ is spatially isomorphic to $\mathscr{A}_{E} \otimes \mathscr{L}(\Re)$ for some Hilbert space $\Re$ [6. Lemma 3 . 12]. On the other hand, the $\mathscr{B} \cap \mathscr{B}^{\prime}$-support of $\bar{E}=I$, so $\mathscr{B}_{E}$ is isomorphic to $\mathscr{B}$ by the condition that $E \in \mathscr{B}^{\prime}$ [3. p. 19]. Therefore $\mathscr{A}$ is isomorphic to $\mathscr{B} \otimes \mathscr{L}(\Re)$.

Remark. Proposition 9 is extended to the following:

If a von Neumann algebra $\mathscr{A}$ is discrete over a von Neumann subalgebra $\mathscr{B}$ containing the center of $\mathscr{A}$, then $\mathscr{A}$ is isomorphic to $(\mathscr{B} \otimes \mathscr{L}(\mathscr{R}))_{E}$, for some Hilbert space $\Re$ and a projection $E$ in $\mathscr{B} \otimes \mathscr{L}(\Omega)$.

The proof is clear from $[3 ;$ p. 58 , Theorem 3$]$. 
The following definition is introduced in [2].

Definition 10. A von Neumann algebra $\mathscr{A}$ is called continuous over a von Neumann subalgebra $\mathscr{B}$ if $\mathscr{A}$ contains no nonzero projections abelian over $\mathscr{B}$.

Proposition 11. Let $\mathscr{B}$ be a von Neumann subalgebra of $\mathscr{A}$. If $\mathscr{A}$ is discrete (resp. continuous) over $\mathscr{B}$, then $\mathscr{A}_{E}$ is discrete (resp. continuous) over $\mathscr{B}_{E}$ for each nonzero $E \in\left(\mathscr{B} \cap \mathscr{B}^{\prime}\right)^{p}$.

Proof. By the definition 10 , it is clear that if $\mathscr{A}$ is continuous over $\mathscr{B}$, then $\mathscr{A}_{E}$ is continuous over $\mathscr{B}_{E}$ for every nonzero $E \in\left(\mathscr{B} \cap \mathscr{B}^{\prime}\right)^{p}$. If $\mathscr{A}$ is discrete over $\mathscr{B}$, there exists a $F \in\left(\mathscr{B}^{c}\right)^{p}$ which is abelian over $\mathscr{B}$ and $\bar{F}=I$. For each nonzero $E \in\left(\mathscr{B} \cap \mathscr{B}^{\prime}\right)^{p}, G=F E$ is abelian over $\mathscr{B}$ by Lemma 4 and

$$
\bar{G}=\overline{F E}=\bar{F} E=E
$$

by Lemma 2 , so that $\mathscr{A}_{E}$ is discrete over $\mathscr{B}_{E}$.

Corollary 12. If $\mathscr{A}$ is an abelian von Neumann algebra, then $\mathscr{A}$ is discrete over a von Neumann subalgebra $\mathscr{B}$ if and only if $\mathscr{B}$ is a type $I$ subalgebra of $\mathscr{A}$ in the sense of Dye [4], that is, each nonzero projection in $\mathscr{A}$ dominates a nonzero projection abelian over $\mathscr{B}$.

Proof. By Theorem 7, the "if" part is clear. Conversely, if there exists a nonzero projection which does not dominate any nonzero projection abelian over $\mathscr{B}$, then we have a nonzero $E \in \mathscr{B}^{p}$ such that $\mathscr{A}_{E}$ is continuous over $\mathscr{B}_{E}$ (cf. [4. p. 124]). By proposition 11 , this is a contradiction.

Proposition 13. Let $\mathscr{B}$ be a von Neumann subalgebra contained in the center of $\mathscr{A}$ and $\left(E_{\iota}\right)_{\iota \in I}$ be an orthogonal family of projections in $\mathscr{B}$ with $\Sigma_{\iota} E_{\iota}=I$. If $\mathscr{A}_{E_{\iota}}$ is discrete (resp. continuous) over $\mathscr{B}_{E_{\iota}}$ for each $\iota$, then $\mathscr{A}$ is discrete (resp. continuous) over $\mathscr{B}$.

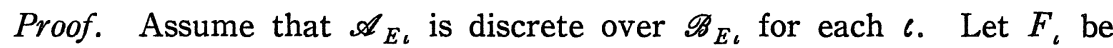
a projection abelian over $\mathscr{B}$ and $\bar{F}_{\iota}=E_{\iota}$, then by the proof of Theorem 7 
$F=\Sigma_{\iota} F_{\iota}$ is abelian over $\mathscr{B}$ and $\bar{F}=\Sigma_{\iota} E_{\iota}=I$. Therefore $\mathscr{A}$ is discrete over $\mathscr{B}$.

Assume that $\mathscr{A}_{E \iota}$ is continuous over $\mathscr{B}_{E \iota}$ for each $\iota$. If $\mathscr{A}$ is not continuous over $\mathscr{B}$, then there exists a nonzero $F \in\left(\mathscr{B}^{c}\right)^{p}$ abelian over $\mathscr{B}$. Let $E_{k}$ be a projection in $\left(E_{\iota}\right)_{\iota \in I}$ such that $F E_{k} \neq 0 . \quad F E_{k}$ is a nonzero projection abelian over $\mathscr{B}$ by Lemma 4 , which contradicts the assumption that $\mathscr{A}_{E_{k}}$ is continuous over $\mathscr{B}_{E_{k}}$.

Theorem 14. Assume that $\mathscr{B}$ is contained in the center of $\mathscr{A}$. Then there exists a unique $E \in \mathscr{B}^{D}$ satisfying the following:

(1) $\mathscr{A}_{E}$ is discrete over $\mathscr{B}_{E}$,

and

(2) $\mathscr{A}_{I-E}$ is continuous over $\mathscr{B}_{I-E}$.

Proof. If $\mathscr{A}$ is not continuous over $\mathscr{B}$, then there exists a nonzero projection in $\mathscr{A}$ abelian over $\mathscr{B}$. Put $E=\sup _{\iota} \bar{E}_{\iota}$, where each $E_{\iota}$ is a nonzero projection abelian over $\mathscr{B}$. It follows that $E \in \mathscr{B}^{p}$ and $\mathscr{A}_{E}$ is discrete over $\mathscr{B}_{E}$. In fact, take a nonzero $P \in \mathscr{B}^{p}$ with $P \leqq E$, then we have $P E_{\iota} \neq 0$ for some $\iota$, which implies $E \geqq P E_{\iota} \neq 0$ and $P E_{\iota} \in\left(\mathscr{B}^{c}\right)^{p}$ because $\mathscr{B}$ is abelian. Since $E_{\iota} \geqq P E_{\iota}, P E_{\iota}$ is abelian over $\mathscr{B}$ by Lemma 4 . Thus by Theorem $7 \mathscr{A}_{E}$ is discrete over $\mathscr{B}_{E}$. If $\mathscr{A}_{I-E}$ is not continuous over $\mathscr{B}_{I-E}$, there exists a nonzero $G \in \mathscr{A}^{p}$ which is abelian over $\mathscr{B}$ and $G \leqq I-E$. By the definition of $E$, we have $G \leqq \bar{G} \leqq E$, which implies that $G=0$. This is a contradiction. Therefore $\mathscr{A}_{I-E}$ is continuous over $\mathscr{B}_{I-E}$.

Assume that, for $G \in \mathscr{B}^{p}, \mathscr{A}_{G}$ is discrete over $\mathscr{B}_{G}$ and $\mathscr{A}_{I-G}$ is continuous over $\mathscr{B}_{I-G}$, then there exists a $Q \in \mathscr{A}^{p}$ which is abelian over $\mathscr{B}$ and $\bar{Q}=G$. By the definition of $E, G \leqq E$. If $E \neq G$, then $E-G$ dominates a nonzero projection abelian over $\mathscr{B}$. On the other hand, $E-G \leqq$ $I-G$. Therefore $I-G$ dominates a nonzero projection abelian over $\mathscr{B}$, which contradicts the assumption that $\mathscr{A}_{I-G}$ is continuous over $\mathscr{B}_{I-G}$. Thus $E=G$, which shows the uniqueness of $E$.

Remark. As a corollary of Theorem 14 , we have the following ( $[3$. p. 121, Cor. 1]); there exists a unique central projection $E$ such that $\mathscr{A}_{E}$ is discrete and that $\mathscr{A}_{I-E}$ is continuous.

3. Let $\mathscr{B}$ be a subalgebra of a von Neumann algebra $\mathscr{A}$. A positive 
linear mapping $e$ of $\mathscr{A}$ onto $\mathscr{B}$ is called an expectaion of $\mathscr{A}$ onto $\mathscr{B}$ if $e$ satisfies the following conditions;

$$
I^{e}=I
$$

and

$$
(A B)^{e}=A^{e} B \text { for all } A \in \mathscr{A} \text { and for all } B \in \mathscr{B}
$$

(cf. [10] and [14]).

The main result in $[2]$ is that concerning $e$-strong Maharam subalgebras:

Definition 15. Let $e$ be a normal expectation of $\mathscr{A}$ onto $\mathscr{B}$. $\mathscr{B}$ is called an e-strong Maharam subalgebra of $\mathscr{A}$ if for any $P \in A^{p}$ and any $B \in \mathscr{B}$ such that $0 \leqq B \leqq P^{e}$ there exists a $Q \in \mathscr{A}^{p}$ such that $Q \leqq P$ and that $Q^{e}=B$.

Theorem A. ([2. Corollary 11]). Let $\mathscr{B}$ be a von Neumann subalgebra of a von Neumann algebra $\mathscr{A}$ contained in the center of $\mathscr{A}$ and $\boldsymbol{e}$ a normal expectation of $\mathscr{A}$ onto $\mathscr{B}$. If $\mathscr{A}$ is continuous over $\mathscr{B}$, then $\mathscr{B}$ is an e-strong Maharam subalgebra of $\mathscr{A}$.

In this section, we shall discuss two von Neumann algebras $\mathscr{C}$ and $\mathscr{A}$ satisfying the following conditions;

$$
\mathscr{C} \supset \mathscr{A} \text { and } \mathscr{Z}=\mathscr{A} \cap \mathscr{A}^{\prime} \supset \mathscr{Z}_{\mathscr{C}}=\mathscr{C} \cap \mathscr{C}^{\prime}
$$

or

$$
\mathscr{C} \supset \mathscr{A} \text { and } \mathscr{Z}=\mathscr{A} \cap \mathscr{A}^{\prime}=\mathscr{C} \cap \mathscr{A}^{\prime} \text {. }
$$

Remark that if $\mathscr{Z}=\mathscr{C} \cap \mathscr{A}^{\prime}$ then $\mathscr{Z} \supset \mathscr{Z}_{\mathscr{G}}$.

Theorem 16. Let $\mathscr{C}$ and $\mathscr{A}$ be two von Neumann algebras satisfying the condition (*). If $\mathscr{C}$ is finite and discrete, then $\mathscr{A}$ is discrete over $\mathscr{Z}_{\mathscr{C}}$.

Proof. If $\mathscr{A}$ is not discrete over $\mathscr{Z}_{\mathscr{C}}$, then there exists by Theorem 14 a nonzero projection $F$ in $\mathscr{Z}_{\mathscr{Q}}$ such that $\mathscr{A}_{F}$ is continuous over $\left(\mathscr{Z}_{\mathscr{C}}\right)_{F}$. $\mathscr{C}_{F}$ and $\mathscr{A}_{F}$ satisfy the condition $(*)$ and $\mathscr{C}_{F}$ is finite and discrete because $F \in\left(\mathscr{Z}_{\mathscr{C}}\right)^{p}$. So we shall assume that $\mathscr{A}$ is continuous over $\mathscr{Z}_{\mathscr{C}}$. 
Since $\mathscr{C}$ is discrete, there exists a projection $E$ in $\mathscr{C}$ such that $\mathscr{C}_{E}=\left(\mathscr{Z}_{\mathscr{B}}\right)_{E}$ and $\mathscr{Z}_{\mathscr{G}}$-support of $E=I$. Since $\mathscr{C}$ is finite, there exists a normal faithful expectation $e$ of $\mathscr{C}$ onto $\mathscr{Z}_{\mathscr{C}}$, that is, canonical natural mapping of $\mathscr{C}$ (cf. [3]). On the other hand, $\mathscr{Z} \supset \mathscr{Z}_{\mathscr{C}}$ and $\mathscr{A}$ is continuous over $\mathscr{Z}_{\mathscr{C}}$. Therefore, by Theorem $A \mathscr{Z}_{\mathscr{C}}$ is an $e$-strong Maharam subalgebra of $\mathscr{A}$, that is, there exists an $F \in \mathscr{A}^{p}$ with $E^{e}=F^{e}$. Applying the comparability theorem to $E$ and $F$, we have a $G \in\left(\mathscr{Z}_{\mathscr{G}}\right)^{p}$ such that $E G<F G$ and that $E(I-G)<F(I-G)$. If $E G \gtrsim F G$, then $E^{e} G \neq F^{e} G$ because $e$ is positive and faithful, which contradicts the property that $E^{e}=F^{e}$. Hence $E G \sim F G$. Similarly, $E(I-G) \sim F(I-G)$.

Therefore $E \sim F$. Since $E$ is abelian over $\mathscr{Z}_{\mathscr{C}}, F$ is abelian over $\mathscr{Z}_{\mathscr{G}}$ cf. [1. Lemma 3]. So that $\mathscr{C}_{F}=\left(\mathscr{Z}_{\mathscr{C}}\right)_{F}$, which implies $\mathscr{A}_{F}=\left(\mathscr{Z}_{\mathscr{C}}\right)_{F}$. Thus $\mathscr{A}$ contains a nonzero projection $F$ abelian over $\mathscr{Z}_{\mathscr{C}}$. This is a contradiction.

Corollary 17. Let $\mathscr{C}$ and $\mathscr{A}$ be two von Neumann algebras satisfying the condition (*). If $\mathscr{C}$ is finite and $\mathscr{A}$ is continuous over $\mathscr{Z}_{\mathscr{G}}$, then $\mathscr{C}$ is continuous.

Proof. If $\mathscr{C}$ is not continuous, there exists a nonzero projection $E$ in $\mathscr{Z}_{\mathscr{G}}$ such that $\mathscr{C}_{E}$ is discrete by Theorem 14 . Then $\mathscr{C}_{E}$ and $\mathscr{A}_{E}$ satisfy the conditions of Theorem 16. Therefore $\mathscr{A}_{E}$ is discrete over $\left(\mathscr{Z}_{\mathscr{G}}\right)_{E}$. Due to Proposition 11, this contradicts with the assumption that $\mathscr{A}$ is continuous over $\mathscr{Z}_{\mathscr{C}}$.

Lemma 18. Let $\mathscr{C}$ and $\mathscr{A}$ be two von Neumann algebras satisfying the condition $(* *)$. If $\mathscr{A}$ is discrete, then $\mathscr{A}=\mathscr{C} \cap \mathscr{Z}^{\prime}$.

Proof. Let $\mathscr{B}$ be a von Neumann algebra generated by $\mathscr{C}^{\prime}$ and $\mathscr{Z}$, then $\mathscr{B}=\left(\mathscr{C} \cap \mathscr{Z}^{\prime}\right)^{\prime}$ and $\mathscr{A}^{\prime} \supset \mathscr{B} \supset \mathscr{Z}$. If $\mathscr{A}$ is discrete, then $\mathscr{A}^{\prime}$ is discrete, cf. $[3$, p. 123, Theorem 1.].

Therefore $\mathscr{B}$ is normal in $\mathscr{A}^{\prime}$ that is,

$$
\mathscr{B}^{c c}=\left(\mathscr{B}^{\prime} \cap \mathscr{A}^{\prime}\right)^{\prime} \cap \mathscr{A}^{\prime}=\mathscr{B},
$$

cf. $\left[3\right.$, p. 307 , exercise 13]. In fact, if $\mathscr{A}^{\prime}$ is discrete, then $\mathscr{A}^{\prime}$ is isomorphic to a von Neumann algebra $\mathscr{D}$ such that $\mathscr{D}^{\prime}$ is abelian, cf. [3]. Let $\mathscr{B} \subset \mathscr{A}^{\prime}$ be isomorphic to $\mathscr{B}_{1} \subset \mathscr{D} . \quad \mathscr{Z} \subset \mathscr{A}^{\prime}$ is isomorphic to $\mathscr{D} \cap \mathscr{D}^{\prime}=\mathscr{D}^{\prime}$. 
Hence $\mathscr{B}_{1} \supset \mathscr{D}^{\prime}$. Therefore

$$
\mathscr{B}_{1}^{c c}=\left(\mathscr{B}_{1}^{\prime} \cap \mathscr{D}\right)^{\prime} \cap \mathscr{D}=\mathscr{B}_{1} \cap \mathscr{D}=\mathscr{B}_{1} .
$$

Therefore $\mathscr{B}^{c c}=\mathscr{B}$.

On the other hand,

$$
\begin{aligned}
\mathscr{B}^{c c} & =\left(\mathscr{B}^{\prime} \cap \mathscr{A}^{\prime}\right)^{\prime} \cap \mathscr{A}^{\prime}=\left(\mathscr{C} \cap \mathscr{Z}^{\prime} \cap \mathscr{A}^{\prime}\right)^{\prime} \cap \mathscr{A}^{\prime} \\
& =\left(\mathscr{Z}^{\prime} \cap \mathscr{Z}\right)^{\prime} \cap \mathscr{A}^{\prime}=\mathscr{Z}^{\prime} \cap \mathscr{A}^{\prime} \\
& =\mathscr{A}^{\prime} .
\end{aligned}
$$

Hence $\mathscr{B}=\mathscr{A}^{\prime}$, that is $\mathscr{A}=\mathscr{B}^{\prime}=\mathscr{C} \cap \mathscr{Z}^{\prime}$.

Lemma 19. Let $\mathscr{C}$ and $\mathscr{A}$ be two von Neumann algebras satisfying the condition (**). If $\mathscr{A}$ is discrete and $\mathscr{C}$ is continuous, then $\mathscr{A}$ is continuous over $\mathscr{Z}_{\mathscr{\varphi}}$.

Proof. If $\mathscr{A}$ is not continuous over $\mathscr{Z}_{\mathscr{E}}$, then there exists a nonzero $E$ in $\mathscr{A}^{p}$ such that $\mathscr{A}_{E}=\left(\mathscr{Z}_{\mathscr{C}}\right)_{E}$, which implies $\mathscr{A}_{E}=\mathscr{Z}_{E}=\left(\mathscr{Z}_{\mathscr{C}}\right)_{E}$.

Since $E \in \mathscr{A} \subset \mathscr{C} \cap \mathscr{Z}^{\prime}$, it follows that

$$
\mathscr{C}_{E}=\mathscr{C}_{E} \cap\left(\mathscr{Z}_{\mathscr{C}}\right)_{E}^{\prime}=\mathscr{C}_{E} \cap \mathscr{Z}_{E}^{\prime}=\left(\mathscr{C} \cap \mathscr{Z}^{\prime}\right)_{E}
$$

On the other hand, $\mathscr{A}$ is discrete, and so $\mathscr{C} \cap \mathscr{Z}^{\prime}=\mathscr{A}$ by Lemma 18 .

Therefore $\mathscr{C}_{E}=\mathscr{A}_{E}$, so that $\mathscr{C}_{E}=\left(\mathscr{Z}_{\mathscr{C}}\right)_{E}$. Thus $\mathscr{C}$ contains a nonzero abelian projection, which is a contradiction.

Theorem 20. Let $\mathscr{C}$ and $\mathscr{A}$ be two von Neumann algebras satisfying the condition $(* *)$. If $\mathscr{C}$ is continuous, then $\mathscr{A}$ is continuous over $\mathscr{Z}_{\mathscr{Q}}$.

Proof. There exists a projection $E$ in $\mathscr{Z}$ such that $\mathscr{A}_{E}$ is discrete and that $\mathscr{A}_{I-E}$ is continuous. Since $\mathscr{Z}_{\mathscr{E}}$ is abelian, it follows that $\mathscr{A}_{I-E}$ is continuous over $\left(\mathscr{Z}_{\mathscr{C}}\right)_{I-E}$, cf. for instance [2. Example 4].

On the other hand, $\mathscr{A}_{E}$ and $\mathscr{C}_{E}$ satisfy the conditions of Lemma 19 because $E \in \mathscr{Z}=\mathscr{C} \cap \mathscr{A}^{\prime}$. Therefore $\mathscr{A}_{E}$ is continuous over $\left(\mathscr{Z}_{\mathscr{C}}\right)_{E}$. So that by an analogy with the proof of Proposition 13, we have $\mathscr{A}$ continuous over $\mathscr{Z}_{\mathscr{C}}$. 
Corollary 21. Let $\mathscr{C}$ and $\mathscr{A}$ be two von Neumann algebras satisfying the conditon $(* *)$. If $\mathscr{A}$ is discrete over $\mathscr{Z}_{\mathscr{C}}$, then $\mathscr{C}$ is discrete.

Proof. If $\mathscr{C}$ is not discrete, there exists a nonzero $E \in\left(\mathscr{Z}_{\mathscr{G}}\right)^{p}$ such that $\mathscr{C}_{E}$ is continuous, by Theorem 14 . Then $\mathscr{C}_{E}$ and $\mathscr{A}_{E}$ satisfy the conditions of Theorem 20 because $E \in \mathscr{Z}_{\mathscr{C}} \subset \mathscr{C} \cap \mathscr{A}^{\prime}$. Therefore $\mathscr{A}_{E}$ is continuous over $\left(\mathscr{Z}_{\mathscr{C}}\right)_{E}$, which contradicts the assumption that $\mathscr{A}$ is discrete over $\mathscr{Z}_{\mathscr{C}}$ by Proposition 11.

Theorem 22. Let $\mathscr{C}$ and $\mathscr{A}$ be two von Neumann algebras satisfying the condition (**). Assume that $\mathscr{C}$ is finite. Then $\mathscr{C}$ is discrete (resp. continuous) if and only if $\mathscr{A}$ is discrete (resp. continuous) over $\mathscr{Z}_{\mathscr{G}}$.

Proof. If $\mathscr{C}$ is discrete, $\mathscr{A}$ is discrete over $\mathscr{Z}_{\mathscr{C}}$ by Theorem 16. If $\mathscr{C}$ is continuous, $\mathscr{A}$ is continuous over $\mathscr{Z}_{\mathscr{C}}$ by Theorem 20 . If $\mathscr{A}$ is discrete over $\mathscr{Z}_{\mathscr{C}}, \mathscr{C}$ is discrete by corollary 21. And if $\mathscr{A}$ is continuous over $\mathscr{Z}_{\mathscr{C}}, \mathscr{C}$ is continuous by corollary 17.

4. Let $G$ be a countable group of $(*-)$ automorphisms of a von Neumann algebra $\mathscr{A}$. An automorphism $\alpha$ of $\mathscr{A}$ is called freely acting on $\mathscr{A}$ when

$$
A B=B^{\alpha} A \quad \text { for all } B \in \mathscr{A}
$$

implies

$$
A=0
$$

([9]). $G$ is called freely acting on $\mathscr{A}$ if $g \neq 1$ (the unit element) in $G$ is freely acting on $\mathscr{A}$.

In this section, we are concerned with a finite von Neumann algebra $\mathscr{A}$ with a faithful normal $G$-invariant trace $\varphi$.

Now, we shall review briefly the concept of crossed product ([11] and [12]). Denote by $\Sigma_{g \in G} g \otimes A_{g}$ an operator valued function on $G$ where $A_{g} \in \mathscr{A}$ is the value of the function at $g \in G$. Let $\mathscr{D}$ be the set of all operator valued functions on $G$ such that $A_{g}=0$ up to a finite subset of $G$. Then $\mathscr{D}$ is a linear space with the usual operation of the addition and scaler multiplication, and becomes a *algebra by the following operations; 


$$
\left(\Sigma_{g \in G} g \otimes A_{g}\right)\left(\Sigma_{h \in G} h \otimes B_{h}\right)=\Sigma_{g, h \in G} g h \otimes A_{g} B_{h}^{g-1}
$$

and

$$
\left(\Sigma_{g \in G} g \otimes A_{g}\right)^{*}=\Sigma_{g \in G} g^{-1} \otimes A_{g}^{* g} .
$$

And $\varphi$ is extended to a faithful trace $\tilde{\varphi}$ on $\mathscr{D}$ by

$$
\tilde{\varphi}\left(\Sigma_{g \in G} g \otimes A_{g}\right)=\varphi\left(A_{1}\right)
$$

Let $\mathfrak{S}$ be the representation space of $\mathscr{A}$ by $\varphi$ (cf. for instance [3]), then $G \otimes \mathfrak{S}$, in the sense of Umegaki [14], is the representation space of $\mathscr{D}$ by $\tilde{\varphi}$. Define operators $I \otimes A$ and $U_{g}(g \in G, A \in \mathscr{A})$ on $G \otimes \mathfrak{E}$ by

$$
I \otimes A\left(\Sigma_{h \in G} h \otimes B_{h}\right)=\Sigma_{h \in G} h \otimes A B_{h}
$$

and

$$
U_{g}\left(\Sigma_{h \in G} h \otimes B_{h}\right)=\Sigma_{h \in G} g h \otimes B_{h}^{g-1}
$$

for any $\Sigma_{h \in G} h \otimes B_{h} \in \mathscr{D}$, where $\mathscr{D}$ is considered as a dense linear subset of $G \otimes \mathfrak{S}$. Then $U_{g}$ is a unitary operator with

$$
U_{g}^{*}(I \otimes A) U_{g}=I \otimes A^{g}
$$

Hereafter, we shall identify $I \otimes A$ with $A$ since $\mathscr{A}$ is isomorphic to $I \otimes \mathscr{A}$.

The crossed product $G \otimes \mathscr{A}$ is the weak closure of $\mathscr{D}$ on $G \otimes \mathfrak{E}$, where $\mathscr{D}$ is now considered as a $*$-algebra of operators on $G \otimes \mathfrak{S}$, that is, a von Neumann algebra generated by $\mathscr{A}$ and $\left\{U_{g}: g \in G\right\}$. Then $G \otimes \mathscr{A}$ is a finite von Neumann algebra with a faithful normal trace $\tilde{\varphi}$.

Haga-Takeda [7] have extended the definition of full group (due to Dye) as in the below and proved Theorem B.

For two automorphisms $\alpha$ and $\beta$ of $\mathscr{A}$, let $F(\alpha, \beta)$ be the maximum central projection such that $\alpha^{-1} \beta$ is an inner automorphism on $\mathscr{A}_{F(\alpha, \beta)}$ (cf. [7] or [9]). Consider the set

$$
[G]=\left\{\text { automorphism } \alpha \text { of } \mathscr{A}: \sup _{g \in G} F(\alpha, g)=I\right\} \text {. }
$$

Then each $\alpha \in[G]$ induces the unitary $U_{\alpha}$ of $G \otimes \mathscr{A}$ with

$$
U_{\alpha}^{*} A U_{\alpha}=A^{\alpha} \quad \text { for } \quad A \in \mathscr{A} \text {. }
$$


$[G]$ forms a group containing $G$ and $G$ is called full if $[G]=G$. By the fixed algebra of $G$, we mean the algebra

$$
\mathscr{Z}(G)=\left\{A \in \mathscr{A} ; A^{g}=A \quad \text { for all } g \in G\right\}
$$

A subalgebra of $G \otimes \mathscr{A}$ containing $\mathscr{A}$ is called an intermediate von Neumann algebra of $G \otimes \mathscr{A}$.

Theorem B ([7. Theorem 2]). Let $G$ be a countable group of automorphisms freely acting on $\mathscr{A}$. Suppose that $\mathscr{A}$ is a finite von Neumann algebra with a faithful normal G-invariant trace. Then the lattice of all intermediate von Neumann algebras $\mathscr{C}$ of $G \otimes \mathscr{A}$ and the lattice of all full subgroups $K$ of $[G]$ are isomorphic by associating with each full subgroup $K$ the intermediate von Neumann subalgebra $\mathscr{C}$

$$
\mathscr{C}=\text { the von Neumann algebra generated by }\left[U_{\alpha} ; \alpha \in K\right]
$$

and with each intermediate von Neumann subalgebra $\mathscr{C}$ the full subgroup $K$

$$
K=\left\{\alpha \in[G] ; U_{\alpha} \in \mathscr{C}\right\}
$$

Now, we shall extend the definition of types of automorphism groups as follows:

Definition 23. A full subgroup $K$ of $[G]$ is called to be discrete type (resp. continuous type) if $\mathscr{A}$ is discrete (resp. continuous) over the fixed algebra $\mathscr{Z}(K)$ of $K$.

Remark that the fixed algebra of a full group is contained in the center of $\mathscr{A}$ because a full group contains all inner automorphisms of $\mathscr{A}$.

Mixed types can occur, but by Theorem 14 a full group $K$ can be devided into purely discrete type and continuous type parts. That is, for the projection $E \in \mathscr{Z}(K)$ in Theorem 14, each of $\mathscr{A}_{E}$ and $\mathscr{A}_{I-E}$ reduces $K$, so that $K$ splits into the direct sum $K_{E}+K_{I-E}$ of two groups, the first a discrete type of automorphisms of $\mathscr{A}_{E}$, the second a continuous type of automorphisms of $\mathscr{A}_{I-E}$. The summands are obviously uniquely determined.

Theorem 24. Let $G$ be a countable group of automorphisms freely acting on $\mathscr{A}$. Suppose that $\mathscr{A}$ is a finite von Neumann algebra with a faithful normal G-invariant trace. Let $\mathscr{C}$ be an intermediate von Neumann 
algebra of $G \otimes \mathscr{A}$ and $K$ a full subgroup of $[G]$ which corresponds to $\mathscr{C}$ in the sense of Theorem $B$. Then $\mathscr{C}$ is discrete (resp. continuous) if and only if $K$ is discrete type (resp. continuous type).

Proof. By the assumption of $G$ and $\mathscr{A}, G \otimes \mathscr{A}$ is finite, and so $\mathscr{C}$ is finite. Furthermore, by [8. Lemma 4.1$]$, we have $\mathscr{C} \cap \mathscr{A}^{\prime}=\mathscr{Z}=\mathscr{A} \cap \mathscr{A}^{\prime}$.

Therefore $\mathscr{C}$ and $\mathscr{A}$ satisfy the conditions of Theorem 22 . On the other hand, $\mathscr{Z}(K)=\mathscr{Z}_{\mathscr{C}}$, the center of $\mathscr{C}$ by [8. Corollary 4.3]. Hence we have this theorem.

In [8], Haga has proved the Dye correspondence in the different form from Theorem 24.

Corollary 25. ([5. Proposition 6.1]). Suppose that $\mathscr{A}$ is abelian, that $G$ is freely acting automorphism group on $\mathscr{A}$ and that $\mathscr{A}$ has a faithful normal $G$-invariant trace. Then the correspondence of intermediate von Neumann subalgebras of $G \otimes \mathscr{A}$ and the full subgroups of $[G]$ in the sense of Theorem $B$ (that is due to Dye [5]) conserves the type.

Especially assume that $\mathscr{A}$ is continuous in Theorem 24 , then $\mathscr{A}$ is continuous over each abelian von Neumann subalgebra of $\mathscr{A}$. Therefore by Theorem 24, we have the following corollary.

Corollary 26. Let $G$ be a countable group of automorphisms freely acting on $\mathscr{A}$. Suppose that $\mathscr{A}$ is a finite continuous von Neumann algebra with a faithful normal $G$-invariant trace. Then each intermediate von Neumann subalgebra of $G \otimes \mathscr{A}$ is continuous.

5. Prof. Y. Nakagami pointed out the following variants of Proposition 9.

Proposition A. Let $\mathscr{A}$ be a finite discrete factor. If $\mathscr{A}$ is discrete over a von Neumann subalgebra $\mathscr{B}$, then $\mathscr{A}$ is isomorphic to $\mathscr{B} \otimes \mathscr{L}(\Re)$ for some Hilbert space $\Re$.

Proof. If $\mathscr{A}$ is discrete over $\mathscr{B}$, then $\mathscr{B}$ is a discrete factor by Proposition 8 and finite. Therefore, $\mathscr{A}$ is isomorphic to $\mathscr{B} \otimes \mathscr{L}(\Re)$ for some Hilbert space $\Omega$. 
Proposition B. Let $\mathscr{A}$ be a von Neumann algebra discrele over a von Neumann subalgebra $\mathscr{B}$ containing the center $\mathscr{Z}$ of $\mathscr{A}$. If $\mathscr{A}$ is properly infinite, then $\mathscr{A}$ is isomorphic to $\Sigma \oplus\left(\mathscr{B}_{E_{\iota}} \otimes \mathscr{L}\left(\mathfrak{E}_{\iota}\right)\right)$, where $E_{\iota}$ runs over a partition of $I$ in $\mathscr{Z}$ and $\mathfrak{C}_{\imath}$ is a Hilbert space for each $\iota$.

Corollary. Let $\mathscr{A}$ be a properly infinite factor discrete over a von Neumann subalgebra $\mathscr{B}$, then $\mathscr{A}$ is isomorphic to $\mathscr{B} \otimes \mathscr{L}(\mathfrak{E})$ for some Hilbert space $\mathfrak{S}$.

Poof of Proposition B. At the first, we shall show that there exists a nonzero $E \in \mathscr{L}^{p}$ such that $\mathscr{A}_{E}$ is isomorphic to $\mathscr{B}_{E} \otimes \mathscr{L}(\mathfrak{g})$ for some Hilbert space $\mathfrak{Q}$.

Since $\mathscr{A}$ is discrete over $\mathscr{B}$, there exists a $F \in\left(\mathscr{B}^{C}\right)^{p}$ such that $\bar{F}=I$ and that $\mathscr{A}_{F}=\mathscr{B}_{F}$. Since there exists a projection $P$ in $\mathscr{Z}$ such that $E P$ is finite and that $F(I-P)$ is properly infinite in $\mathscr{A}$, we may assume that $F$ is finite or properly infinite. Let $\left\{F_{\iota} ; \iota \in I\right\}$ be a maximal family of equivalent and mutually orthogonal projections in $\mathscr{A}$ such that $F=F$, for some $\iota \in I$. Then there exists a nonzero central projection $E$ in $\mathscr{A}$ satisfying

$$
\left(I-\Sigma_{\iota \in I} F_{\iota}\right) E<F E \text {. }
$$

If $F$ is finite and $I$ is finite, then the central projection

$$
E=\sum_{\iota \in I} F_{\iota} E+\left(I-\sum_{\iota \in I} F_{\iota}\right) E
$$

is finite, which contradicts that $\mathscr{A}$ is properly infinite. If $F$ is properly infinite, for each $F_{\iota}$, there exists a family of countable projections in $\mathscr{A}$ which are equivalent to $F_{\iota}$. Therefore we may choose $I$ as an infinite set. Since

$$
E=\Sigma_{\iota \in I} F_{\imath} E+\left(I-\Sigma_{\iota \in I} F_{\imath}\right) E<\Sigma_{\iota \in I} F_{\imath} E \leqq E,
$$

it follows that $E \sim \sum_{\iota \in I} F_{\iota} E$. Therefore $\mathscr{A}_{E}$ is spatially isomorphic to $\mathscr{A}_{F E} \otimes \mathscr{L}(\mathfrak{E})$ for some Hilbert space $\mathscr{S}$ with $\operatorname{dim}$. $\mathfrak{Q}=$ card. $I$. Since $\overrightarrow{F E}=E, \mathscr{B}_{E}$ is isomorphic to $\mathscr{B}_{F E}=\mathscr{A}_{F E}$. Thus there exists a nonzero projection $E$ in $\mathscr{Z}$ such that $\mathscr{A}_{E}$ is isomorphic to $\mathscr{B}_{E} \otimes \mathscr{L}(\mathfrak{C})$.

Let $\left(E_{\iota}\right)_{\iota \in I}$ be a maximal orthogonal family of projections in $\mathscr{Z}$ such

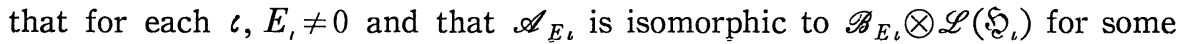


Hilbert space $\mathfrak{S}_{\iota}$. If $\sum_{\iota \in I} E_{\iota} \neq I$, then $G=I-\sum_{\iota \in I} E_{\iota}$ is a nonzero projection in $\mathscr{Z}$. By the assumption, $\mathscr{A}_{G}$ is properly infinite and $\mathscr{A}_{G}$ is discrete over $\mathscr{B}_{G}$ by Proposition 11 . Therefore, there exists a nonzero projection $Q$ in $\mathscr{Z}$ such that $Q \leqq G$ and that $\mathscr{A}_{Q}$ is isomorphic to $\mathscr{B}_{Q} \otimes \mathscr{L}(\Re)$ for some Hilbert space $\mathfrak{R}$, which contradicts the maximality of $\left\{E_{\iota}\right\}$. Hence $\Sigma_{\iota \in I} E_{\iota}=I$, that is, $\mathscr{A}$ is isomorphic to $\Sigma_{\iota \in I} \oplus \mathscr{A}_{E_{\iota}}=\Sigma_{\iota \in I} \oplus\left(\mathscr{B}_{E_{\iota}} \otimes \mathscr{L}\left(\mathfrak{E}_{\iota}\right)\right)$.

\title{
References
}

[1] Choda, M., Abelian projections over a von Neumann subalgebra, Proc. Iapan Acad., 48 (1972), 384-388.

[2] Choda, M., A von Neumann algebra continuous over a von Neumann subalgebra, Proc. Japan Acad., 49 (1973), 174-178.

[3] Dixmier, J., Les algèbres d'opérateurs dans l'espace Hlibertien, Gauthier-Villars, Paris (1957).

[4] Dye, H. A., On groups of measure preserving transformations I, Amer. J. Math., 81 (1959), 119-159.

[5] Dye, H. A., On groups of measure preserving transformations, II, Amer. J. Math, 85 (1963), 551-576.

[6] Murray, F.J., and J. von Neumann, On rings of operators IV, Ann. Math., 44 (1943), 716-808.

[7] Haga, Y., and Z. Takeda, Correspondence between subgroups and subalgebras in a cross product von Neumann algebra, Tohoku Math. J., 24 (1972), 167-190.

[8] Haga, Y., On subalgebras of cross product von Neumann algebra, Preprint.

[9] Kallman, R.R., A generalization of free action, Duke Math. J., 36 (1969), 781-789.

[10] Nakamura M., and T. Turumaru, Expectations in an operator algebra, Tohoku Math. J., 6 (1954), 182-188.

[11] Nakamura M., and Z. Takeda, On some elementary properties of the crossed product of von Neumann algebras, Proc. Japan Acad., 34 (1958), 489-494.

[12] Turumaru, T., Crossed product of operator algebras, Tohoku. Math. J., 10 (1958), $355-365$.

[13] Umegaki, H., Conditional expectation in an operator algebra, Tohoku Math. J., 6 (1954), 177-181.

[14] Umegaki, H., Positive definite functions and direct product of Hilbert space, Tohoku Math J., 7. (1955), 201-211.

\author{
M. Choda \\ Department of Mathematics \\ Osaka Kyoiku University \\ Tennoji, Osaka
}

